\title{
On Connectivity Indices of an Infinite Family of The Linear Parallelogram of Benzenoid Graph
}

\author{
Mohammad Reza Farahani \\ ${ }^{1}$ Department of Applied Mathematics, Iran University of Science and Technology (IUST), \\ Narmak, Tehran 16844, Iran \\ E-mail address: Mr_Farahani@mathdep.iust.ac.ir, MrFarahani88@gmail.com
}

Keywords: Molecular graph, Connectivity Indices, Molecular Graph, Benzenoid Graph.

ABSTRACT. A topological index of a graph $G$ is a numeric quantity related to $G$ which is describe molecular graph G. In this paper the Atom Bond Connectivity (ABC) and Geometric-Arithmetic (GA) indices of an infinite class of the linear parallelogram of benzenoid graph.

\section{INTRODUCTION}

Mathematical chemistry is a branch of theoretical chemistry using mathematical methods to discuss and predict molecular properties without necessarily referring to quantum mechanics [1-5]. Molecular descriptors play significant role in chemistry, pharmacology, etc. Among them, topological indices have a prominent place [1]. A topological index of a graph $G$ is a numeric quantity related to $\mathrm{G}$ which is describe molecular graph $\mathrm{G}$

One of the best known and widely used is the connectivity index $\chi$ introduced in 1975 by Milan Randić [6]. Estrada et al. proposed a new index, known as the atom-bond connectivity index $(A B C)$. This index is defined as $[7,8]$

$$
A B C(G)=\sum_{u v \in E(G)} \sqrt{\frac{d_{v}+d_{u}-2}{d_{v} \times d_{u}}}
$$

where the summation goes over all edges of G, $d_{u}$ and $d_{v}$ are the degrees of the terminal vertices $u$ and $v$ of edge $\mathrm{e}=u v$ and $E(G)$ is the edge set of $\mathrm{G}$ with cardinality $|E(G)|$.

Another topological indices namely, Geometric-Arithmetic index $(G A(G))$ introduced by D.Vukicevic and B.Furtula [9-13], respectively and was defined as follows:

$$
G A(G)=\sum_{u v \in E(G)} \frac{2 \sqrt{d_{v} d_{u}}}{d_{v}+d_{u}}
$$

\section{MAIN RESULTS AND DISCUSSSION}

Consider the molecular graph linear parallelogram of benzenoid graph $P(n, m), \forall m, n \in \mathbb{N}-\{1\}$. In generally consider the linear parallelogram benzenoid graph $P(n, m)$ depicted in Figure 1 and see [14-22].

In this section, we compute the Atom Bond Connectivity (ABC) and Geometric-Arithmetic (GA) indices of an infinite family of the linear parallelogram of benzenoid graph $P(n, m)$.

Theorem 1. Consider the linear parallel ogramof benzenoid graph $P(n, m)(\forall m, n \in \mathbb{N}-\{1\})$. Then the Atom Bond Connectivity index $\operatorname{ABC}(P(n, m))$ is equal to:

$$
A B C(P(n, m))=2 m n-2(2 / 3-\sqrt{ } 2)(m+n)+2(1-\sqrt{ } 2)
$$

Proof of Theorem 1. Let $P(n, m)$ be the linear parallelogram of benzenoid graph $(\forall m, n \in \mathbb{N}-\{1\})$ depicted in Figure 1. This benzenoid graph has exactly $2 m n+2 m+2 n$ vertices/atoms and 
$3 m n+2 n+2 m-1$ edges/bonds. From structure of the linear parallelogram of benzenoid graph, one can see that all vertices of $P(n, m)$ have degree two or three. So we can divide its vertex and edge sets $V(P(n, m))$ and $E(P(n, m))$ of $G$ to following partitions as:

$$
\begin{gathered}
V_{3}=\left\{v \in V(G) \mid d_{v}=3\right\}, \\
V_{2}=\left\{v \in V(G) \mid d_{v}=2\right\} \\
E_{4}=E_{4} *=\left\{u v \in E(G) \mid d_{u}=d_{v}=2\right\}, \\
E_{5}=E_{6} *=\left\{u v \in E(G) \mid d_{u}=2 \& d_{v}=3\right\}, \\
E_{6}=E_{9} *=\left\{u v \in E(G) \mid d_{u}=d_{v}=3\right\}
\end{gathered}
$$

From Figure 1 and [21], it is easy to see that

$\left|V_{2}\right|=m+n+1+m+n+1=2(m+n+1)$ and $\left|V_{3}\right|=2 m n-2$.

$\left|E_{4}\right|=\left|E_{4} *\right|=\left|\left\{u v \in E(P(n, m)) \mid d_{u}=d_{v}=2\right\}\right|=4$.

$\left|E_{5}\right|=\left|E_{6}{ }^{*}\right|=\left|\left\{u v \in E(P(n, m)) \mid d_{u}=2 \& d_{v}=3\right\}\right|=2(m-1)+2(n-1)+2(m-1)+2(n-1)=4(m+n-2)$.

$\left|E_{6}\right|=\left|E_{9} *\right|=\left|\left\{u v \in E(P(n, m)) \mid d_{u}=d_{v}=3\right\}\right|=3 m n+2 n+2 m-1-4(m+n-2)-4=3 m n-2 n-2 m+3$.

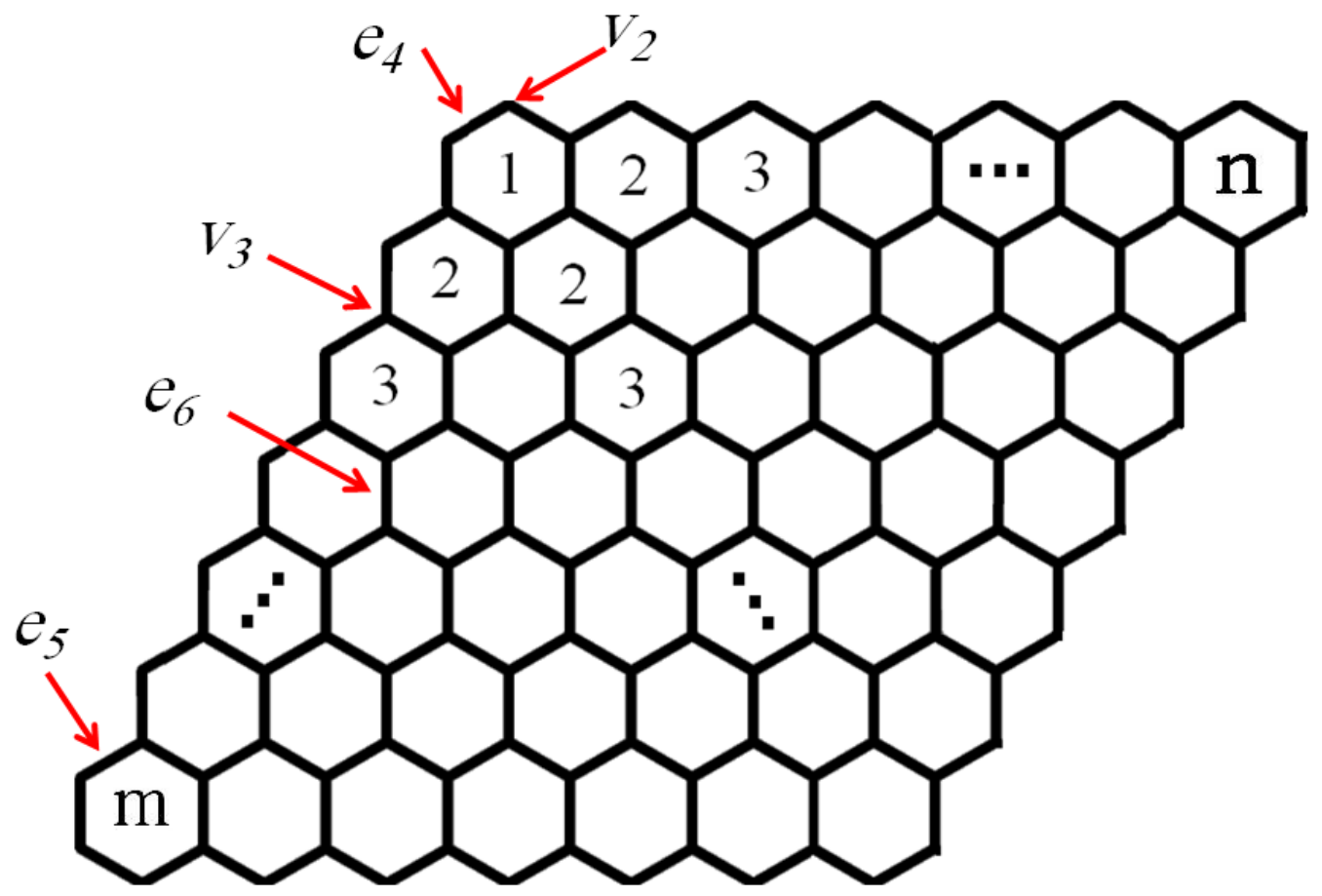

Fig. 1. The linear parallelogram of benzenoid graph $P(n, m), \forall m, n \in \mathbb{N}$. [21]

Now by using above results, we start to compute the Atom Bond Connectivity index for the linear parallelogram of benzenoid graph as:

$$
\begin{aligned}
A B C(P(n, m)) & =\sum_{u v \in E(P(n, m))} \frac{\sqrt{d_{v}+d_{u}-2}}{\sqrt{d_{v} \times d_{u}}} \\
& =\sum_{u v \in E_{9}^{*}} \frac{\sqrt{d_{v}+d_{u}-2}}{\sqrt{d_{v} \times d_{u}}}+\sum_{u v \in E_{6}^{*}} \frac{\sqrt{d_{v}+d_{u}-2}}{\sqrt{d_{v} \times d_{u}}}+\sum_{u v \in E_{4}^{*}} \frac{\sqrt{d_{v}+d_{u}-2}}{\sqrt{d_{v} \times d_{u}}}
\end{aligned}
$$




$$
\begin{aligned}
& =\sum_{u v \in E_{9}^{*}} \frac{\sqrt{3+3-2}}{\sqrt{3 \times 3}}+\sum_{u v \in E_{6}^{*}} \frac{\sqrt{3+2-2}}{\sqrt{3 \times 2}}+\sum_{u v \in E_{4}^{*}} \frac{\sqrt{2+2-2}}{\sqrt{2 \times 2}} \\
& =\frac{2}{3}\left|E_{9}^{*}\right|+\frac{\sqrt{2}}{2}\left|E_{6}^{*}\right|+\frac{\sqrt{2}}{2}\left|E_{4}^{*}\right| \\
& =2 / 3 \times(3 m n-2 n-2 m+3)+\frac{\sqrt{2}}{2} \times[4(m+n-2)+4] \\
& =2 m n-2(2 / 3-\sqrt{ } 2) m-2(2 / 3-\sqrt{2}) n+2(1-\sqrt{ } 2) \\
& =2 m n-2(2 / 3-\sqrt{ } 2)(m+n)+2(1-\sqrt{ } 2) .
\end{aligned}
$$

Theorem 2. $\forall m, n \in \mathbb{N}-\{1\}$, let $P(n, m)$ be the linear parallelogram of benzenoid graph. Then the Geometric-Arithmetic index $G A(P(n, m))$ is equal to

$$
G A(P(n, m))=3 m n+2\left(\frac{2 \sqrt{6}}{5}-1\right)(m+n)+\left(7-\frac{16 \sqrt{6}}{5}\right)
$$

Proof of Theorem 2. $\forall m, n \in \mathbb{N}-\{1\}$, Consider the linear parallelogram of benzenoid graph $P(n, m)$. By using above mention proof, we can compute the Geometric-Arithmetic index of $P(n, m)$ easily as follows:

$$
\begin{aligned}
G A(P(n, m)) & =\sum_{u v \in E(P(n, m))} \frac{2 \sqrt{d_{v} d_{u}}}{d_{v}+d_{u}} \\
& =\sum_{u v \in E_{9}^{*}} \frac{2 \sqrt{3 \times 3}}{3+3}+\sum_{u v \in E_{6}^{*}} \frac{2 \sqrt{3 \times 2}}{3+2}+\sum_{u v \in E_{4}^{*}} \frac{2 \sqrt{2 \times 2}}{2+2} \\
& =\left|E_{9} *\right|+\frac{2 \sqrt{6}}{5}\left|E_{6} *\right|+\left|E_{4} *\right| \\
& =(3 m n-2 n-2 m+3)+\frac{2 \sqrt{6}}{5} \times 4(m+n-2)+(4) \\
& =3 m n+2\left(\frac{2 \sqrt{6}}{5}-1\right) m+2\left(\frac{2 \sqrt{6}}{5}-1\right) n+\left(7-\frac{16 \sqrt{6}}{5}\right) \\
& =3 m n+2\left(\frac{2 \sqrt{6}}{5}-1\right)(m+n)+\left(7-\frac{16 \sqrt{6}}{5}\right) . \mathbf{\square}
\end{aligned}
$$

\section{CONCLUSION}

A topological index of a graph $G$ is a numeric quantity related to $G$ which is describe molecular graph G. In this paper the Atom Bond Connectivity (ABC) and Geometric-Arithmetic (GA) indices of an infinite class of the linear parallelogram of benzenoid graph.

\section{References}

[1] N. Trinajstić, Chemical Graph Theory, CRC Press, Boca Raton, FL, (1992).

[2] R. Todeschini, V. Consonni, Handbook of Molecular Descriptors, Wiley-VCH, Weinheim, (2000).

[3] I. Gutman, O. E. Polansky, Mathematical Concepts in Organic Chemistry, Springer Verlag, Berlin, 1986.

[4] N. Trinajstić, I. Gutman, Croat. Chem. Acta, 2002, 75, 329-356. 
[5] A. Graovac, I. Gutman, N. Trinajstić, Topological Approach to the Chemistry of Conjugated Molecules, Springer Verlag, Berlin, 1977.

[6] M. Randic, On the characterization of molecular branching.. J. Amer. Chem. Soc. 97, 66096615, (1975).

[7] E. Estrada, L. Torres, L. Rodriguez and I. Gutman. Indian J. Chem. 37, 849 (1998)

[8] K.Ch. Das, K. Xu and A. Graovac. Maximal Unicyclic Graphs With Respect to New Atombond Connectivity Index. Acta Chim. Slov. 2013, 60, 34-42.

[9] D. Vukicevic and B. Furtula. Topological index based on the ratios of geometrical and arithmetical means of end-vertex degrees of edges. J. Math. Chem.46, 1369-1374. (2009).

[10]L. Xiao, S. Chen, Z. Guo and Q. Chen. The Geometric-Arithmetic Index of Benzenoid Systems and Phenylenes Int. J. Contemp. Math. Sciences. 5, (45), 2225-2230, (2010).

[11]M. Ghorbani and M. Ghazi, Computing some topological indices of Triangular Benzenoid. Digest. J. Nanomater. Bios, 5(4), (2010), 1107-1111.

[12]M.R. Farahani. Some Connectivity Indices and Zagreb Index of Polyhex Nanotubes. Acta Chim. Slov. 59, 779-783 (2012).

[13] M.R. Farahani. Computing some connectivity indices of Nanotubes. Adv. Mater. Corrosion. 1, (2012) 57-60.

[14]P.V. Khadikar. Padmakar-Ivan Index in Nanotechnology. Iranian Journal of Mathematical Chemistry, 2010, 1(1), 7-42.

[15]M. Alaeiyan, R. Mojarad and J. Asadpour. A new method for computing eccentric connectivity polynomial of an infinite family of linear polycene parallelogram benzenoid. Optoelectron. Adv. Mater.-Rapid Commun. 2011, 5(7), 761-763.

[16]M. Alaeiyan and J. Asadpour. Computing the MEC polynomial of an infinite family of the linear parallelogram $P(n, n)$. Optoelectron. Adv. Mater.-Rapid Commun. 2012, 6(1-2), 191 193.

[17] M.R. Farahani. Computing a Counting polynomial of an infinite family of linear polycene parallelogram benzenoid graph $P(a, b)$. Journal of Advances in Physics, 3(1), (2013), 186190.

[18] M.R. Farahani. On Sadhana polynomial of the linear parallelogram $P(n, m)$ of benzenoid graph. Journal of Chemica Acta. 2(2), (2013), 95-97.

[19]M.R. Farahani. Computing the Omega polynomial of an infinite family of the linear parallelogram $P(n, m)$. Journal of Advances in Chemistry. 1, (2013), 106-109.

[20]M.R. Farahani. Connective Eccentric Index of Linear Parallelogram $P(n, m)$. Int. Letters of Chemistry, Physics and Astronomy. 18, (2014), 57-62.

[21]M.R. Farahani. Two Types of Connectivity indices of the linear parallelogram benzenoid. New Frontiers in Chemistry. 23(1), (2014), 73-77.

[22] M.R. Farahani. Zagreb indices and their polynomials of the linear parallelogram of benzenoid graph. Global Journal of Chemistry. 1(1), 2015, 16-19. 